\section{Solar surfaces: A bad idea or tomorrow's mainstream application?- ADDENDUM}

doi: https://doi.org/10.1557/mre.2019.11

The following footnote should be included in this article [1]:

This paper was commissioned for publication by David Cahen, who served as Editor-in-Chief of this journal from 2014-2018.
Sarah Kurtz

REFERENCE:

1. Kurtz S. (2019). Solar surfaces: A bad idea or tomorrow's mainstream application? MRS Energy \& Sustainability, 6, E8. Cambridge University Press. 